\title{
Brief Advice on Smoking Reduction Versus Abrupt Quitting for Smoking Cessation in Chinese Smokers: A Cluster Randomized Controlled Trial
}

\author{
Man Ping Wang' ', William H. Li', Yee Tak Cheung ${ }^{12}$, Oi Bun Lam', Yongda Wu', \\ Antonio C. Kwong ${ }^{3}$, Vienna W. Lai ${ }^{3}$, Sophia S. Chan', Tai Hing Lam²
}

${ }^{1}$ School of Nursing, University of Hong Kong; ${ }^{2}$ School of Public Health, University of Hong Kong; ${ }^{3}$ Hong Kong Council on Smoking and Health

Corresponding Author: Man Ping Wang, PhD, School of Nursing, University of Hong Kong, Pokfulam, Hong Kong, China. Telephone: 852-39176636; Fax: 852-28726079; E-mail: mpwang@hku.hk

\begin{abstract}
Aims: To compare the efficacy of brief advice about cut-down-to-quit (CDTQ) with that of brief advice about quit immediately (Ol), as delivered by trained volunteers, without the use of pharmacological therapy, to outreach-recruited Chinese smokers in Hong Kong who intend to quit smoking.

Methods: Smokers $(N=1077)$ who enrolled in the Quit and Win Contest 2014 and intended to quit or reduce smoking were randomized in participation sessions to CDTQ $(n=559)$ and QI $(n=518)$ groups. Subjects in the CDTQ group received brief advice and a card about smoking reduction. Subjects in the Ql group received brief advice and a leaflet about quitting smoking. All received a smoking cessation booklet and corresponding CDTQ or QI brief telephone advice at intervals of 1 week, 1 month, or 2 months. The primary outcomes were self-reported 7-day point prevalence abstinence (PPA) at the 3-month and 6-month follow-ups. The secondary outcomes included abstinence rate as validated by biochemical tests, smoking reduction ( $\geq 50 \%$ reduction from baseline), and quit attempt (QA). The outcome assessors were blinded as to group assignment.

Results: By intention to treat, the QI and CDTQ groups showed similar results as regards (a) selfreported PPA (10.6\% [95\% Cl 8.1\%-13.6\%] vs. 9.1\% [95\% Cl 6.9\%-11.8\%]), (ii) validated abstinence rate $(5.6 \%[3.8 \%-7.9 \%]$ vs. $5.4 \%[3.6 \%-7.6 \%]$ ), and (iii) $\mathrm{QA}$ rate $(59.2 \%$ [53.5\%-64.8\%] vs. $54.1 \%$ $[48.7 \%-59.3 \%]$ ) at 6-month. However, the CDTQ group showed a significantly higher reduction rate than the Ql group (20.9\% [Cl 17.6\%-24.5\%] vs. 14.5\% [11.6\%-17.8\%]). The overall intervention adherence was suboptimal (45.4\%), particularly in the CDTQ group (42.3\%). Self-efficacy as regards quitting of smoking was similar between the groups at 6 months.

Conclusions: Brief advice on CDTQ and QI had similar short-term PPAs. Longer-term follow-up is needed to understand the latent effect of smoking reduction on abstinence.

Implications:This is the first randomized controlled trial in ethnic Chinese smokers to evaluate the relative efficacy of brief advice on (a) CDTO and (b) QI as regards quitting. The two interventions showed similar effects as regards PPA. The findings suggested that brief advice on CDTQ may be as effective as brief advice on $\mathrm{Ql}$ in smokers recruited in community settings.
\end{abstract}




\section{Introduction}

Cut-down-to-quit (CDTQ) is an alternative approach to quitting abruptly. It envisages reducing the number of cigarettes gradually to achieve total abstinence. The CDTQ approach is controversial as regards its efficacy in achieving harm reduction and smoking cessation. Meta-analysis studies found similar quit rates between CDTQ and quit immediately (QI) interventions in smokers with or without intention to quit smoking. ${ }^{1,2}$ Subgroup analysis of limited nonpharmacologic studies of the meta-analysis showed similar quit rates in CDTQ and QI groups in smokers who wanted to quit smoking. ${ }^{1}$ Two recent randomized controlled trials (RCT) found that, among smokers without intention to quit smoking, CDTQ intervention without medication yielded similar point prevalence abstinence (PPA) results as compared with other QI or motivational counseling, but better PPA than no intervention or usual care. ${ }^{3,4}$ The smoking cessation effects of CDTQ intervention combined with nicotine replacement therapy (NRT) differed by whether there was initial intention to quit. ${ }^{1,5,6}$ In two meta-analysis studies, when CDTQ was combined with NRT (with or without behavioral support) it doubled the sustained quit rate in smokers who had no initial intention to quit ${ }^{5}$ but produced only a similar sustained quit rate in smokers who had expressed an intention to quit. ${ }^{1}$ Another recent RCT on NRT-assisted CDTQ in smokers who attended general practitioner clinics and were willing to quit found significantly lower PPA for the CDTQ intervention as compared with QI (relative risk 0.71, 95\% CI 0.46-0.91) at the 6-month follow-up. ${ }^{6}$

Most CDTQ studies have been carried out in Western subjects. Only one trial has been conducted on Chinese subjects. ${ }^{7}$ Our earlier RCT found that smoking reduction counseling combined with NRT was more effective than brief advice on QI as assessed by PPA rates at the 6 -month follow-up $(17.0 \%$ vs. $10.2 \%, p=.01)$ in Chinese smokers who had not expressed an intention to quit. ${ }^{8}$ Subsequent posteriori analysis of the same study found that smoking reduction counseling was more effective in achieving total abstinence in hardcore smokers than in non-hardcore smokers, ${ }^{9}$ and that a greater percentage of smoking reduction predicted abstinence. ${ }^{10}$ Smoking reduction intervention may be particularly important for countries where smoking prevalence is decreasing in the population, and hardcore smokers predominate. Hong Kong is a typical example of such a situation, in particular after the implementation of strong smoke-free legislation and other tobacco control measures. ${ }^{11}$ Many reduction trials have involved pharmacologic therapies. It remains uncertain whether smoking reduction counseling alone is effective in smokers who are willing to quit or reduce smoking. Unlike earlier trials that used intensive smoking reduction consultation, we aimed to test a short brief advice on reduction to achieve smoking abstinence among Chinese smokers in Hong Kong.

\section{Methods}

\section{Design}

This cluster randomized controlled trial (cRCT) recruited Chinese adult smokers in Hong Kong. The subjects were randomized to receive brief advice at recruitment sessions about either CDTQ or QI for smoking cessation. At baseline, the CDTQ group received brief advice and a card on smoking reduction; thereafter, on follow-up, they received brief smoking reduction advice over the telephone. The QI group received brief QI advice and a QI card, and brief smoking cessation advice over the telephone at follow-up. Both groups received a 12-page self-help booklet on smoking cessation. Please refer to Supplementary Material for a brief description of self-help booklet and cards. The primary outcomes were self-reported PPA values for the past 7 days, at 3-month and 6-month follow-ups.

\section{Subjects}

The subjects were smokers who joined the Quit and Win Contest in 2014. The contest provided incentives to promote quitting of smoking: the details of the recruitment procedure have been published elsewhere. ${ }^{12}$ In brief, trained smoking cessation ambassadors actively recruited adult smokers from the community in all 18 districts of Hong Kong. Smokers who had smoked at least 1 cigarette daily in the past 3 months, had an expiratory carbon monoxide (CO) concentration of at least $4 \mathrm{ppm}$ and expressed a willingness to reduce or quit smoking were eligible to participate in the trial. We excluded smokers who were non-Chinese, of ages $<18$ or who had participated in other smoking cessation programs. The sample size was estimated based on the assumption of $5 \%$ significant level, $80 \%$ power and the finding from the earlier CDTQ trial which showed an odds ratio/effect size of $1.67 .{ }^{8}$ Assuming a $60 \%$ retention rate and intra-cluster correlation coefficient of 0.01 , the sample size was calculated as 1252 smokers.

\section{Randomization}

Cluster randomization based on recruitment sessions $(N=67)$ was used as onsite individual randomization would be subject to contamination. All subjects recruited from the same session were randomly allocated to either the CDTQ group or the QI group, using permuted block randomization. The primary investigator, who was not involved in the recruitment, randomly generated blocks, with each block size being equal to 4 and containing a random permutation of the 2 groups. All the blocks were combined to generate the list of group allocation. The recruitment staff was informed about the group allocation one day prior to the recruitment session. The subjects were not informed about the intervention in other groups. Outcome assessors and statistical analysts were blinded as to the group allocation.

\section{Interventions}

Subjects in the CDTQ group received brief advice (about 5 minutes) on smoking reduction. The briefing used the AWARD model ${ }^{13}$ which includes (a) Ask about smoking history, (b) Warn about the health risk (that one in every two smokers would die of smoking-related causes), (c) Advise to quit by cutting down cigarette consumption at their own pace within 3 months, (d) Refer the smokers to a smoking cessation clinic, and (e) Do it again by repeating the intervention during each telephone follow-up and encouraging smokers who relapse to restart smoking reduction. The smoking cessation ambassadors helped the subjects to set strategies for gradual reduction: progressively reduce cigarettes smoked per day by $25 \%$ in the first week, $50 \%$ in the first month, $75 \%$ in the second month and quit altogether in the third month ${ }^{14}$; use a scheduled reduction approach by increasing time intervals between each cigarette, ${ }^{15}$ or a hierarchical reduction approach starting with the easiest cigarette of the day to forgo and moving to the hardest cigarette to give up (or vice versa). ${ }^{16}$ Subjects also received a smoking reduction card containing the above information. Subjects in the CDTQ group who planned to quit within 7 days were encouraged to quit and received a reduction card if they would like to reduce smoking after the 7-day. Those who 
failed to quit in 7-day would receive the same CDTQ brief advice through telephones at 1-week follow-up. Smokers who quit within 7 days were encouraged during follow-ups to remain abstinent. The subjects also received brief 'booster' messages (1-2 minutes) over the telephone to reinforce the reduction advice at 1 week, 1 month, and 2 months. The subjects in the QI group received AWARD advice (about 5 minutes) focusing on $\mathrm{QI}^{13}$ and our ambassadors encouraged them to set a quit day close to baseline. A QI card and brief QI booster messages over the telephone were also provided as for the CDTQ group. At baseline, both groups were given a standard 12-page smoking cessation booklet designed by the Hong Kong Council on Smoking and Health (COSH). ${ }^{17}$

\section{Measures and Statistical Analysis}

Based on interviews, each subject completed a questionnaire on sociodemographic characteristics, smoking and quit history, nicotine dependence as measured by Heaviness of Smoking Index (HSI), ${ }^{18}$ and perceived self-efficacy (difficulties, importance assigned and confidence) as regards quitting of smoking. ${ }^{19,20}$ The primary outcomes were measured in terms of the self-reported past-7-day PPA at the 3-month and 6-month telephone follow-ups. The secondary outcomes included biochemically validated (salivary cotinine $<10 \mathrm{ng}$ / $\mathrm{mL}$ ) abstinence rate, smoking reduction ( $\geq 50 \%$ reduction from baseline), and QA (any abstinence attempt lasting 24 hours or longer after participating in the project). Logistic regression was used to calculate odds ratios (ORs) of outcomes between the CDTQ and QI groups, using intention to treat (ITT) and complete case analysis. We also compared group prevalences of indicators of intervention compliance, which included whether the subjects read the booklet (ever vs. never), read the education cards (ever vs. never) and followed the instructions (yes vs. no).

\section{Ethical Approval}

The study was approved by the Institutional Review Broad of the University of Hong Kong/Hospital Authority West Cluster (UW 14-382) and registered in trial registry (NCT02539875).

\section{Results}

Of 1328 smokers assessed for eligibility, 1077 were selected and randomized to CDTQ $(n=559)$ and QI $(n=518)$ groups. Retention rates were $65.3 \%$ in the CDTQ group and $60.8 \%$ in the QI group at 3 months follow-up, and fell to $63.9 \%$ and $58.7 \%$, respectively, at 6 months (Figure 1). The subjects were $42( \pm 15.5)$ years of age on average; $81.4 \%$ were male subjects; $83.8 \%$ had at least a secondary school education; $55.8 \%$ had monthly family income $<\mathrm{HK} \$ 20000$ (US\$2564); and $75.5 \%$ were employed. Both groups shared very similar smoking and quitting histories, which included average age at which smoking started, $\mathrm{CO}$ concentration, proportions of quit and reduction attempts in the last 12-month, and self-perceived efficacy as regards quitting (Table 1). The CDTQ group had a significantly higher proportion of subjects in paid employment, higher daily cigarette consumption and higher nicotine dependence level (HSI score $\geq 4$ ).

Self-reported PPA was nonsignificantly lower in the CDTQ group than in the QI group at 3 months $(7.0 \%$ vs. $10.0 \%$; OR $0.67,95 \%$ CI $0.44-1.04)$ and 6 months $(9.1 \%$ vs. $10.6 \%$; OR $0.85,95 \%$ CI $0.57-1.26)$ (Table 2). Biochemically validated abstinence rates were also nonsignificantly lower in the CDTQ group $(3.8 \%)$ than in the QI group $5.6 \%$ (OR $0.6395 \%$ CI $0.36-1.12$ ) at 3 months,

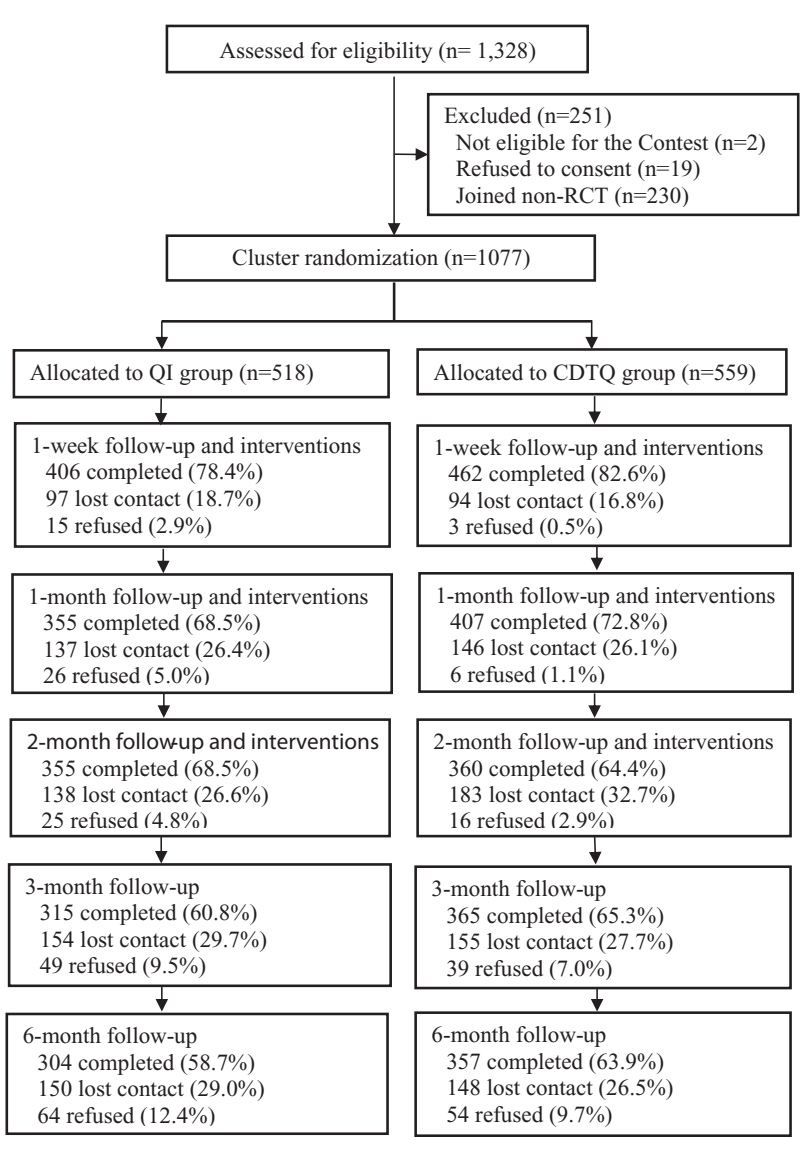

Figure 1. Trial flow chart.

and this difference dipped at 6 -months $(5.4 \%$ vs. $5.6 \%$; OR 0.96 , $95 \%$ CI $0.57-1.62)$. Smoking reduction $(\geq 50 \%)$ rates progressively increased in the CDTQ group between the 1-month and 6-month follow-ups, whereas they gradually reduced in the QI group during the same period. The absolute difference in point prevalence of smoking reduction between the CDTQ and QI groups increased from $-7.1 \%$ at 1 month to $6.5 \%$ at 6 months. The smoking reduction rate was significantly higher in the CDTQ group $(20.9 \%)$ than in the QI group (14.5\%) at the 6-month follow-up (OR 1.56, 95\% CI 1.14-2.15). Past QA rates (defined as QA after joining the project) were similar between the two groups at follow-up. The results remained robust after adjusting for demographic characteristics and baseline HSI.

The rates of use of booklets $(63.2 \%)$ and education cards $(73.2 \%)$ were satisfactory (Table 3 ) but less than half $(45.4 \%)$ of the subjects were adherent to the suggested quitting methods. Subjects in the CDTQ group $(42.3 \%$ ) had a marginally significant lower rate of compliance to the suggested quitting methods than subjects in the QI group $(49.0 \%)$ at 6 months $(p=.08)$. The subjects' levels of selfperceived efficacy in quitting smoking were similar between groups.

\section{Discussion}

This cRCT has a larger sample size compared to earlier trials that were included in a meta-analysis on the effectiveness of smoking reduction advice and behavioral support on smoking abstinence. ${ }^{1}$ We found similar self-reported PPA values, validated abstinence rates and QA between the CDTQ and QI groups of ethnic Chinese 
Table 1. Baseline Subject Characteristics

\begin{tabular}{|c|c|c|c|}
\hline & CDTQ $(n=559)$ & $\mathrm{QI}(n=518)$ & $p$ value \\
\hline Male & 82.6 & 80.1 & .27 \\
\hline Mean age $( \pm S D)$, year & $42.8 \pm 14.7$ & $42.6 \pm 16.4$ & .86 \\
\hline Secondary education or above & 84.1 & 83.6 & .84 \\
\hline In-paid employment & 78.3 & 72.6 & .03 \\
\hline Living in rental public housing & 43.5 & 45.4 & .52 \\
\hline Monthly household income <HKD 20000 (7.8 = 1USD) & 53.9 & 57.8 & .21 \\
\hline Mean age of starting smoking $( \pm S D)$, year & $17.6 \pm 5.9$ & $17.5 \pm 6.0$ & .98 \\
\hline Mean daily cigarette consumption $( \pm S D)$ & $16.2 \pm 9.5$ & $14.7 \pm 8.5$ & .01 \\
\hline Mean expired CO concentration $( \pm S D)$, ppm & $20.0 \pm 12.8$ & $19.3 \pm 13.0$ & .21 \\
\hline Higher nicotine dependence (HSI score $\geq 4$ ) & 38.5 & 32.2 & .03 \\
\hline No previous quit attempt & 27.2 & 24.7 & .37 \\
\hline Any quit attempt(s) in the last 12 months & 23.1 & 27.3 & .11 \\
\hline Any smoking reduction attempt(s) in the last 12 months & 29.5 & 32.1 & .37 \\
\hline \multicolumn{4}{|l|}{ Self-efficacy on quitting $(\text { mean } \pm S D)^{a}$} \\
\hline Perceived difficulty & $7.4 \pm 2.4$ & $7.2 \pm 2.5$ & .41 \\
\hline Perceived confidence & $5.6 \pm 2.4$ & $5.8 \pm 2.3$ & .31 \\
\hline Perceived importance & $7.8 \pm 2.2$ & $7.8 \pm 2.3$ & .62 \\
\hline
\end{tabular}

$\mathrm{CDTQ}=$ cut-down-to-quit; $\mathrm{CO}$ = carbon monoxide; HSI = Heaviness of Smoking Index; QI = quit immediately.

${ }^{a}$ Score $0-10$, higher score indicates higher levels of perception.

Table 2. Smoking Cessation Outcomes

\begin{tabular}{|c|c|c|c|c|c|}
\hline & CDTQ, $n(\%)$ & QI, $n(\%)$ & Absolute difference (95\% CI), \% point & Odds ratio $(95 \% \mathrm{CI})$ & Adjusted odds ratio ${ }^{a}(95 \%$ CI) \\
\hline \multicolumn{6}{|c|}{ Self-report abstinence } \\
\hline 1-month & $26(4.7)$ & $43(8.3)$ & $-3.7(-6.6$ to -0.7$)$ & $0.54(0.33 \text { to } 0.89)^{* *}$ & $0.59(0.35 \text { to } 0.96)^{*}$ \\
\hline 2-month & $30(5.4)$ & $58(11.2)$ & $-5.8(-9.1$ to -2.5$)$ & $0.45(0.28 \text { to } 0.71)^{* * *}$ & $0.45(0.28 \text { to } 0.73)^{* * *}$ \\
\hline 3-month & $39(7.0)$ & $52(10.0)$ & $-3.1(-6.4$ to 0.3$)$ & $0.67(0.44$ to 1.04$)$ & $0.71(0.45$ to 1.11$)$ \\
\hline 6-month & $51(9.1)$ & 55 (10.6) & $-1.5(-5.1$ to 2.1$)$ & $0.85(0.57$ to 1.26$)$ & $0.87(0.57$ to 1.32$)$ \\
\hline \multicolumn{6}{|c|}{ Validated abstinence ${ }^{\mathrm{b}}$} \\
\hline 3-month & $21(3.8)$ & $29(5.6)$ & $-1.8(-4.4$ to 0.7$)$ & $0.63(0.36$ to 1.12$)$ & $0.72(0.40$ to 1.29$)$ \\
\hline 6-month & $30(5.4)$ & $29(5.6)$ & $-0.2(-3.0$ to 2.5$)$ & $0.96(0.57$ to 1.62$)$ & $0.99(0.58$ to 1.70$)$ \\
\hline \multicolumn{6}{|c|}{ Smoking reduction by $50 \%$} \\
\hline 1-month & $107(19.1)$ & $136(26.3)$ & $-7.1(-12.1$ to -2.1$)$ & $0.49(0.36 \text { to } 0.67)^{* * * * *}$ & $0.50(0.36 \text { to } 0.70)^{* * * *}$ \\
\hline 2-month & $103(18.4)$ & $119(23.0)$ & $-4.5(-9.4$ to 0.3$)$ & $0.67(0.49 \text { to } 0.94)^{*}$ & $0.71(0.50 \text { to } 0.99)^{*}$ \\
\hline 3-month & $136(24.3)$ & $113(21.8)$ & $2.5(-2.5$ to 7.5$)$ & 0.95 (0.68 to 1.32$)$ & $1.00(0.72$ to 1.41$)$ \\
\hline 6-month & $117(20.9)$ & $75(14.5)$ & $6.5(1.9$ to 11.0$)$ & $1.56(1.14 \text { to } 2.15)^{* * *}$ & $1.54(1.11 \text { to } 2.14)^{* * *}$ \\
\hline \multicolumn{6}{|c|}{ Quit attempt ${ }^{c}$} \\
\hline 3-month & $117(32.1)$ & $124(39.4)$ & $-7.3(-14.5$ to -0.1$)$ & $0.73(0.53 \text { to } 1.00)^{* *}$ & $0.78(0.56$ to 1.08$)$ \\
\hline 6-month & $142(39.8)$ & $125(41.1)$ & $-1.3(-8.9$ to 6.2$)$ & $0.95(0.69$ to 1.29$)$ & $1.07(0.77$ to 1.49$)$ \\
\hline
\end{tabular}

CDTQ = cut-down-to-quit; CI = confidence interval; HIS = Heaviness of Smoking Index; QI = quit immediately.

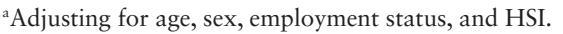

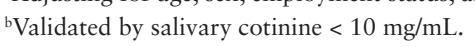

'Excluded quitters.

$* p<.05 ; * p<.01 ; * * p<.001$.

daily smokers at follow-ups at 3 months and 6 months. The findings were different from our earlier trial in which CDTQ plus NRT was found to be effective in helping to achieve abstinence among smokers who had not been ready to quit smoking. ${ }^{8}$ A meta-analysis also found that CDTQ plus medication was more effective in boosting abstinence among smokers who were not ready to quit. ${ }^{7}$ Our findings, however, were consistent with the systematic reviews on CDTQ plus either medication or nonpharmacologic interventions such as counseling and behavioral support. ${ }^{1,2}$ Smoking reduction is commonly adopted by habitual smokers and brief advice is often offered by smoking cessation services providers (eg, quitlines) in Hong Kong and elsewhere..$^{21}$ Our findings suggest that, in smokers who intend to quit smoking, brief advice on CDTQ may have similar effects on quitting as advice on QI. CDTQ may be particularly useful for smokers who are unwilling to quit or have had difficulties in quitting. Smoking reduction may improve health and increase the likelihood of success in quitting in the future. ${ }^{22}$

A possible explanation for the similarity in quit rates between the CDTQ and QI groups could be that CDTQ requires an understanding of the reduction strategies and a proper monitoring of the number of cigarettes consumed or reduced. These may be difficult to follow for elderly smokers and those with lower education levels. This theory is supported by our further subgroup analyses which showed that old age $(9.1 \%$ aged $\geq 65$ years $)$ and education only up to primary level $(16.2 \%)$ were associated with low odds of smoking reduction and cessation in the CDTQ group at follow-up (data not shown in table), although the interaction effects of age and education on the outcomes were not statistically significant. More than 
Table 3. Intervention Adherence and Self-Efficacy on Quitting at 6-Month

\begin{tabular}{|c|c|c|c|c|}
\hline & Total $(N=661)$ & CDTQ $(n=357)$ & $\mathrm{QI}(n=304)$ & $p$ value \\
\hline \multicolumn{5}{|l|}{ Booklet } \\
\hline Ever use & $418(63.2)$ & $225(63.0)$ & $193(63.5)$ & .90 \\
\hline Perceived usefulness $(\text { mean } \pm S D)^{\mathrm{a}}$ & $3.0 \pm 0.9$ & $2.9 \pm 0.8$ & $3.1 \pm 0.9$ & .05 \\
\hline \multicolumn{5}{|l|}{ Education card } \\
\hline Ever use & $484(73.2)$ & $271(75.9)$ & $213(70.1)$ & .09 \\
\hline Perceived usefulness $(\text { mean } \pm S D)^{\mathrm{a}}$ & $3.3 \pm 0.8$ & $3.2 \pm 0.8$ & $3.3 \pm 0.8$ & .69 \\
\hline \multicolumn{5}{|l|}{ Self-efficacy on quitting $(\text { mean } \pm S D)^{a}$} \\
\hline Perceived difficulty & $7.3 \pm 2.0$ & $7.5 \pm 2.0$ & $7.0 \pm 2.0$ & .01 \\
\hline Perceived confidence & $5.5 \pm 2.5$ & $5.4 \pm 2.5$ & $5.6 \pm 2.5$ & .31 \\
\hline Perceived importance & $7.6 \pm 2.0$ & $7.7 \pm 1.9$ & $7.6 \pm 2.0$ & .74 \\
\hline
\end{tabular}

CDTQ = cut-down-to-quit; ITT = intention to treat; QI = quit immediately.

${ }^{\text {aS }}$ core ranges from 1 to 5 with a higher score indicates usefulness.

${ }^{\mathrm{b}}$ Score ranges from 0 to 10 with higher score indicates a high level.

$70 \%$ of the subjects in the CDTQ group did read and refer to the education card. The adherence rate to the suggested quitting strategies was marginally significantly lower in the CDTQ group (42.3\%) than in the QI group (49.0\%). Another explanation for these findings could be that the brief advice given to the subjects in the CDTQ group was not comprehensive enough to deliver an understanding of the reduction strategies, and that these may require more detailed explanations as compared to the advice given to subjects in the QI group. On the other hand, brief advice is a more feasible intervention than intensive counseling for recruiting smokers in community settings (eg, shopping malls, streets and housing estate public areas, etc.) and intensive counseling may be unacceptable to some smokers.

Smoking reduction may require a longer time to practice and move toward achieving abstinence. We observed that the smoking reduction rate gradually increased between the 1-month and 6-month follow-ups in the CDTQ group but declined during the same time line in the QI group. This suggests that smokers in the CDTQ group may require more than a 6-month period to practice the strategies to reduce cigarette consumption $\geq 50 \%$ and to achieve abstinence. Our previous trial using NRT to help gradual cessation in smokers found that smoking reduction predicted subsequent abstinence, ${ }^{10}$ which was consistent with a recent UK study which showed that smokers who used NRT consciously attempted to reduce cigarette consumption. ${ }^{23}$ Longer-term studies, such as those involving 1-year follow-up, are needed to evaluate the effectiveness of CDTQ in achieving smoking abstinence.

This study had several limitations. First, the discrepancy between self-reported PPA and validated abstinence rates suggested that selfreported PPA might be overestimated as a result of a lack of evidence about which group was particularly affected. However, validated abstinence rates were very similar between CDTQ and QI groups $(5.4 \%$ vs. $5.6 \%)$, suggesting that the conclusions were unlikely to have been affected by the overestimated self-reported abstinence. Second, smoking reduction was not validated by biochemical tests, such as exhaled $\mathrm{CO}$ or saliva cotinine. However, the validity of biochemical tests on smoking reduction has not been well established, particularly among heavy smokers. ${ }^{24}$ Third, $38.7 \%$ of the subjects were lost by the 6-month follow-up. Although the proportions of loss to follow-up were similar in both groups and the use of intention-to-treat might reduce the bias, the findings should be interpreted with caution. Fourth, although the small proportion of female smokers $(18.6 \%)$ in our study reflected the general prevalence of female smokers in Hong Kong, the results may not be generalizable to Western countries where female smoking is more common. Given that all the subjects were enrolled to win prizes in the Quit and Win Contest, the findings of this trial may not be directly comparable to other smoking reduction trials.

\section{Conclusions}

Brief advice on CDTQ and QI achieved similar short-term PPA in smokers enrolled in the 2014 Quit and Win Contest in Hong Kong. A longer-term follow-up is needed to understand the latent effect of smoking reduction in the CDTQ group on abstinence.

\section{Supplementary Material}

Supplementary data are available at Nicotine \& Tobacco Research online.

\section{Funding}

The Quit and Win Contest in 2014 was funded by COSH.

\section{Declaration of Interests}

None declared.

\section{Acknowledgments}

We would like to thank the subjects for participating in the trial, and staff from $\mathrm{COSH}$ and $\mathrm{HKU}$ smoking cessation team for project management.

\section{References}

1. Lindson-Hawley N, Aveyard P, Hughes JR. Reduction versus abrupt cessation in smokers who want to quit. Cochrane Database Syst Rev. 2012;11:CD008033.

2. Stead LF, Lancaster T. Interventions to reduce harm from continued tobacco use. Cochrane Database Syst Rev. 2007;(3):CD005231.

3. Meyer C, Ulbricht S, Haug S, et al. Motivating smokers to quit using computer-generated letters that target either reduction or cessation: a population-based randomized controlled trial among smokers who do not intend to quit. Drug Alcohol Depend. 2016;166:177-186.

4. Klemperer EM, Hughes JR, Solomon LJ, Callas PW, Fingar JR. Motivational, reduction and usual care interventions for smokers 
who are not ready to quit: a randomized controlled trial. Addiction. 2017;112(1):146-155.

5. Moore D, Aveyard P, Connock M, Wang D, Fry-Smith A, Barton P. Effectiveness and safety of nicotine replacement therapy assisted reduction to stop smoking: systematic review and meta-analysis. BMJ. 2009;338:b1024.

6. Lindson-Hawley N, Banting M, West R, Michie S, Shinkins B, Aveyard P. Gradual versus abrupt smoking cessation: a randomized, controlled noninferiority trial. Ann Intern Med. 2016;164(9):585-592.

7. Wu L, Sun S, He Y, Zeng J. Effect of smoking reduction therapy on smoking cessation for smokers without an intention to quit: an updated systematic review and meta-analysis of randomized controlled. Int J Environ Res Public Health. 2015;12(9):10235-10253.

8. Chan SS, Leung DY, Abdullah AS, Wong VT, Hedley AJ, Lam TH. A randomized controlled trial of a smoking reduction plus nicotine replacement therapy intervention for smokers not willing to quit smoking. Addiction. 2011;106(6):1155-1163.

9. Lam TH, Cheung YT, Leung DY, Abdullah AS, Chan SS. Effectiveness of smoking reduction intervention for hardcore smokers. Tob Induc Dis. 2015;13(1):9.

10. Cheung YT, Lam TH, Leung DY, Abdullah AS, Chan SS. Nicotine replacement therapy to aid gradual cessation in smokers with no intention to quit: association between reduction quantity and later abstinence. Prev Med Rep. 2015;2:196-201.

11. Leung DY, Chan SS, Chan V, Lam TH. Hardcore smoking after comprehensive smoke-free legislation and health warnings on cigarette packets in Hong Kong. Public Health. 2016;132:50-56.

12. Wang MP, Li WH, Jiang N, et al. E-cigarette awareness, perceptions and use among community-recruited smokers in Hong Kong. PLoS One. 2015;10(10):e0141683.

13. Chan SS, Wong DC, Cheung YT, et al. A block randomized controlled trial of a brief smoking cessation counseling and advice through short message service on participants who joined the Quit to Win Contest in Hong Kong. Health Educ Res. 2015;30(4):609-621.
14. Hughes JR, Solomon LJ, Livingston AE, Callas PW, Peters EN. A randomized, controlled trial of NRT-aided gradual vs. abrupt cessation in smokers actively trying to quit. Drug Alcohol Depend. 2010;111(1-2):105-113.

15. Cinciripini PM, Wetter DW, McClure JB. Scheduled reduced smoking: effects on smoking abstinence and potential mechanisms of action. Addict Behav. 1997;22(6):759-767.

16. Flaxman J. Quitting smoking now or later: gradual, abrupt, immediate and delayed quitting. Behavior Therapy. 1978;9:260-270.

17. Hong Kong Council on Smoking and Health. The First Step "Quit Smoking”. http://www.smokefree.hk/UserFiles/resources/about_us/books/ Smoking\%20cessation\%20booklet_2014.pdf. Accessed 11 June, 2016.

18. Borland R, Yong HH, O'Connor RJ, Hyland A, Thompson ME. The reliability and predictive validity of the Heaviness of Smoking Index and its two components: findings from the International Tobacco Control Four Country study. Nicotine Tob Res. 2010;12(suppl):S45-S50.

19. Rollnick S. Readiness, Importance, and Confidence: Critical Conditions of Change in Treatment. 1998.

20. Lam TH, Abdullah AS, Chan SS, Hedley AJ; Hong Kong Council on Smoking and Health Smoking Cessation Health Centre (SCHC) Steering Group. Adherence to nicotine replacement therapy versus quitting smoking among Chinese smokers: a preliminary investigation. Psychopharmacology (Berl). 2005;177(4):400-408.

21. Asfar T, Ebbert JO, Klesges RC, Klosky JL. Use of smoking reduction strategies among U.S. tobacco quitlines. Addict Behav. 2012;37(4):583-586.

22. Aveyard P, Lindson-Hawley N, Hastings G, de Andrade M. Should smokers be advised to cut down as well as quit? BMJ. 2014;348:g2787.

23. Lindson-Hawley N, Shinkins B, West R, Michie S, Aveyard P. Does cigarette reduction while using nicotine replacement therapy prior to a quit attempt predict abstinence following quit date? Addiction. 2016;111(7):1275-1282.

24. Godtfredsen NS, Prescott E, Vestbo J, Osler M. Smoking reduction and biomarkers in two longitudinal studies. Addiction. 2006;101(10):1516-1522. 